\title{
Dynamical characteristics of microvascular networks with a myogenic response gradient
}

Anastasiia Neganova ${ }^{1}$

${ }^{1}$ Department of Biomedical Sciences, Copenhagen University, Copenhagen, Denmark

\section{Abstract}

Purpose: Myogenic response is the ability of smooth muscle cells lining the vascular wall to react to changing intravascular pressure: increasing pressure normally induces contraction whereas decreasing pressure leads to dilatation. Experimental studies show that the intensity of the myogenic response is different in arteriolar vessels of different radii: smaller arterioles react relatively more intensely, but over a more narrow range of pressures, than larger arterioles. In a network of vessels, this gives rise to a myogenic response gradient. The physiological significance of this gradient is, nonetheless, debated. Our purpose is to investigate the dynamical characteristics of microvascular networks with a myogenic response gradient by means of mathematical modeling.

Methods: We present a mathematical vascular network model which includes a detailed description of vessel wall mechanics and the myogenic response gradient. We focus on the influence of this gradient on short-term network dynamics. We perform a series of numerical simulations in both symmetrical and asymmetrical vascular trees in which the individual vessel is given a realistic morphology, i.e., relative wall thickness is smaller in larger vessels.

Results: Our main findings show that the presence of a myogenic response gradient:

1. adjusts flow and pressure in the capillary bed to an adequate level and dampens oscillations transmitted from upstream feeding vessels;

2. provides the network as a whole with a basal level of tone necessary for the operation of vasomotor mechanisms other than the myogenic response; and

3. provides the system with the overall ability to autoregulate network flow smoothly.

Correspondence: Department of Biomedical Sciences, Copenhagen University, Blegdamsvej 3, 2200 Copenhagen N, Denmark.

E-mail: anastasiia@sund.ku.dk 
Conclusion: The mathematical model shows that networks with a myogenic response gradient present advantages regarding the physiological function of regulating flow in a bifurcating network compared to networks without myogenic response and passive networks.

Keywords: mathematical modeling, myogenic response, myogenic response gradient, oscillations, vascular network

\section{Introduction}

In recent years, mathematical modeling has become a useful tool for gaining a deeper understanding of the physiological processes that affect blood flow regulation in vascular networks. A combined description of vascular wall mechanics and network structure that allows for subsequent simulation of the system may reveal the dynamical features of such networks rather than just characterizing their steady-state behaviour.

Myogenic response is the reaction of the smooth muscle cells (SMC) encircling the blood vessel wall to a pressure stimulus. It is normally seen as vessel wall contraction in response to increasing intraluminal pressure and relaxation in response to decreasing pressure. ${ }^{1,2}$ Myogenic activity depends on stress-induced depolarization of the SMC membrane. ${ }^{3}$ Calcium influx through voltage-operated calcium channels (VOCC) and an increase in cytosolic calcium concentration activate the contractile apparatus, eventually leading to a change in vessel diameter. ${ }^{4}$ The myogenic response is observed in most vascular beds, with the reaction to a given pressure step typically being inversely proportional to vessel size. ${ }^{5,6}$ Variations in myogenic responsiveness are observed not only in vessels of different diameter, but also in vessels of the same diameter but belonging to different vascular beds. ${ }^{3,7}$

A variety of mathematical implementations of the myogenic response ${ }^{8-11}$ and its gradient throughout a vascular network ${ }^{12}$ have been published. However, the shortterm dynamical behaviour of a network with a myogenic gradient has, to the best of our knowledge, not previously been subject to study.

A microvascular network can be modeled in different ways. A simple approach is to model a lattice of hexagons. This particular geometrical structure has been used to investigate the role of wall shear stress in network remodeling. ${ }^{13,14}$ Vascular networks can also be described as a circuit of resistors, with vessels in different parts of the network lumped into compartments on the basis of vessel type. ${ }^{15}$ Another common approach is to model a network as a bifurcating tree. The advantage in this case is a somewhat greater similarity with the structure of a real microvascular network. ${ }^{14,16-18}$ Alternatively, the vascular tree structure can be derived directly from experimental data. ${ }^{19,20}$

A gradient in myogenic responsiveness seems to be an ubiquitous property of microvascular networks. ${ }^{5}$. As a logical extension of previous studies, we therefore propose a short-term dynamical model of a vascular network that features detailed me- 
chanics of the vessel wall ${ }^{21}$ as well as a myogenic response gradient throughout the network. We address the following questions:

1. How does a myogenic response gradient influence short-term microvascular flow and pressure regulation?

2. Having such a gradient, how does symmetry versus asymmetry of the network structure influence network autoregulation?

\section{Mathematical model}

\subsection{Mechanics of the vascular wall}

The mechanical model of the vascular wall has been adapted from VanBavel and Tuna $^{21}$ and modified. Model elements applied in the present simulations are summarized below. All model parameters are listed in Table 1 and Table 2 (see Appendix).

As in previous papers, ${ }^{11,21,22}$ we introduce three types of stress:

1. Passive stress, $\sigma_{p}$, is generated by the connective tissue of the vascular wall, which consists of collagen and elastin fibers. ${ }^{22}$ It depends exponentially on the tissue strain, $\varepsilon$ :

$$
\sigma_{p}=C_{p 1} \cdot\left(e^{a_{p 1} \cdot \varepsilon}-1\right)+C_{p 2} \cdot\left(e^{a_{p 2} \cdot \varepsilon}-1\right),
$$

where $C_{p 1}, C_{p 2}, a_{p 1}$, and $a_{p 2}$ are constants of the passive stress-strain curve.

2. Active stress, $\sigma_{a}$, is a stress generated by contractile activity of the SMC. It is proportional to the tone, $\Psi$, and the maximal capacity for generating active stress, $\sigma_{a m}$ :

$$
\begin{gathered}
\sigma_{a}=\Psi \cdot \sigma_{a m}, \\
\sigma_{a m}=\sigma_{a m_{o p t}} \cdot e^{-\left(\frac{l_{r e f}-l_{a o p t}}{b_{a}}\right)^{2}},
\end{gathered}
$$

where $\sigma_{a m_{o p t}}$ is the peak value of $\sigma_{a m}$ at the SMC-length $l_{a o p t}, b_{a}$ is a constant, and $l_{\text {ref }}$ is the length of the cell.

3. Cytoskeletal stress, $\sigma_{c}$, is a stress generated by the cytoskeleton during elongation of the SMC. It prevents the cell from being stretched excessively:

$$
\sigma_{c}=\sigma_{c 0} \cdot \frac{e^{b_{c} \frac{l_{r e f}-l_{c 0}}{l_{c 0}}}-e^{-b_{c}}}{1-e^{-b_{c}}},
$$

where $\sigma_{c 0}$ is a cytoskeletal stress at SMC length $l_{c 0}$ and $b_{c}$ is a constant of the SMC length-cytoskeletal stress curve.

It is assumed that these three types of stress affect the vascular wall in equal proportion:

$$
C S A_{i}=f_{i} \cdot w C S A
$$


where $i=p, a, c$ defines the passive, active, and cytoskeletal types of stress, respectively. The weighting coefficient for each stress type is $f_{i}$. CSA $A_{i}$ is a portion of the wall cross-sectional area, $w C S A$, which is affected by the particular type of stress. ${ }^{21}$

Hence, the mean wall stress, $\sigma$, consists of three components:

$$
\sigma=\sigma_{p} \cdot f_{p}+\sigma_{a} \cdot f_{a}+\sigma_{c} \cdot f_{c} .
$$

The equilibrium wall stress, $\bar{\sigma}$, is:

$$
\bar{\sigma}=P \cdot r_{i} / h,
$$

where $h=w C S A / 2 \pi r_{m}$ is the wall thickness with $r_{i}=r_{m}-h / 2$, where $r_{m}$ and $r_{i}$ are midwall and inner radii, respectively.

The degree of stretching of the vessel wall is expressed as the strain, $\varepsilon$. In this particular case, the strain is calculated as a relative change in midvessel radius from its unloaded state, $r_{m \text {, slack }}$, (i.e., the state when SMC are fully deactivated and transmural pressure is equal to zero):

$$
\varepsilon=\frac{r_{m}-r_{m, \text { slack }}}{r_{m, \text { slack }}} .
$$

In a state of mechanical equilibrium, when the stress generated by the different components of the wall, $\sigma$ (Eq. 6 ), is equal to the equilibrium wall stress, $\bar{\sigma}$ (Eq. 7 ), the vessel radius does not change and $\varepsilon$ is constant. When mechanical equilibrium is disturbed for any reason, the time evolution of the strain, $\varepsilon$, is calculated as:

$$
\frac{d \varepsilon}{d t}=k_{1} \cdot(\bar{\sigma}-\sigma),
$$

where $k_{1}$ is a time constant for this process.

The vessel wall tone, $\Psi$, is defined as the ratio of actual active stress and maximal active stress. The tone is 0 when the vessel is fully relaxed, i.e., when the active stress is 0 , and 1 when the contractile activity of the wall SMC is maximal, i.e., when the actual active stress is equal to the maximal active stress. As opposed to the previous formulation, ${ }^{21}$ our model does not include any external constrictors and dilators, hence, the tone is caused only by myogenic activation of the vessel wall, depending in turn on the wall stress. The interaction of the equilibrium tone, $\bar{\Psi}$, and the equilibrium wall stress, $\bar{\sigma}$, is described by means of a Hill-type equation:

$$
\bar{\Psi}=\frac{\bar{\sigma}^{h c}}{\bar{\sigma}^{h c}+\sigma_{50} h c},
$$

where $\sigma_{50}$ is the stress at half-maximal activation and $h c$ is the Hill coefficient.

The temporal evolution of the tone is described as:

$$
\frac{d \Psi}{d t}=k_{2} \cdot(\bar{\Psi}-\Psi)
$$


where $k_{2}$ is a time constant.

Although myogenic reactivity is most pronounced in small muscular arteries and arterioles, venous vessels normally possess some tone and may react weakly to changes in pressure. ${ }^{23,24}$ On the other hand, capillaries are normally considered non-contractile because of the lack of SMC coating. In some cases, however, pericytes covering the outer surface of the vessel may provide some weak contractility. ${ }^{25}$ Collectively, therefore, myogenic reactivity is modeled only explicitly in arterial-side vessels, whereas venoules and capillaries are modeled as having a constant, weak tone equal to 0.05 and 0.01 , respectively.

In a microvascular network, larger upstream vessels typically display myogenic responsiveness over a broarder pressure range, but react less forcefully to a given pressure step compared to smaller downstream vessels. The smallest precapillary vessels may have a relatively weak myogenic response, nonetheless. ${ }^{5}$. A myogenic response gradient that mimics this experimentally observed behaviour was introduced into the model by modifying model parameters which define the shape of passive, active, and cytoskeletal stresses as well as equilibrium tone. We introduce the dependencies of these parameters on initial vessel radius, $r_{i 0}$, according to the following equations:

$$
\begin{gathered}
C_{p 1}^{\prime}=\frac{C_{p 1}}{f u n c_{1}\left(r_{i 0}\right)}, C_{p 2}^{\prime}=\frac{C_{p 2}}{\text { func }_{1}\left(r_{i 0}\right)}, \\
\sigma_{a m_{o p t}}^{\prime}=\frac{\sigma_{a m_{o p t}}}{\text { func }_{1}\left(r_{i 0}\right)}, \\
\sigma_{c 0}^{\prime}=\frac{\sigma_{c 0}}{f u n c_{1}\left(r_{i 0}\right)}, \\
\bar{\Psi}=\text { func }_{2}\left(r_{i 0}, \bar{\sigma}^{h c}\right) \cdot \frac{\bar{\sigma}^{h c}}{\bar{\sigma}^{h c}+\sigma_{50} h c},
\end{gathered}
$$

where $f u n c_{1}\left(r_{i 0}\right)$ and $\mathrm{func}_{2}\left(r_{i 0}\right)$ are approximating functions (Appendix). Hereafter, these modified parameters are used in Equations (1), (2), (3), (4), and (10) for network simulations.

\subsection{Network properties}

In the present formulation, a symmetrical network is a bifurcating network where each vessel within a given generation has the same length and radius, and where the venous side is a topological reflection of the arterial side with vessels having the same lengths but larger radii. In contrast, an asymmetrical network is a network where two daughter vessels arising from a stem at a given branch point may neither have the same lengths nor the same radii. In this case, however, the venous side is a topological reflection of the arterial side, with corresponding vessels having the same lengths, but larger radii. 
Experimental data ${ }^{26}$ was used in order to find relations between parent and daughter vessel diameters, as well as between the length and diameter of the individual vessel for the human retina. These relations are summarized in Figure 1 and are further described in Appendix Equations (17-19). We then use a probability-based algorithm to generate the network structure as proposed by Postnov et al. ${ }^{27}$ with the necessary modifications to create the venous part of the tree.
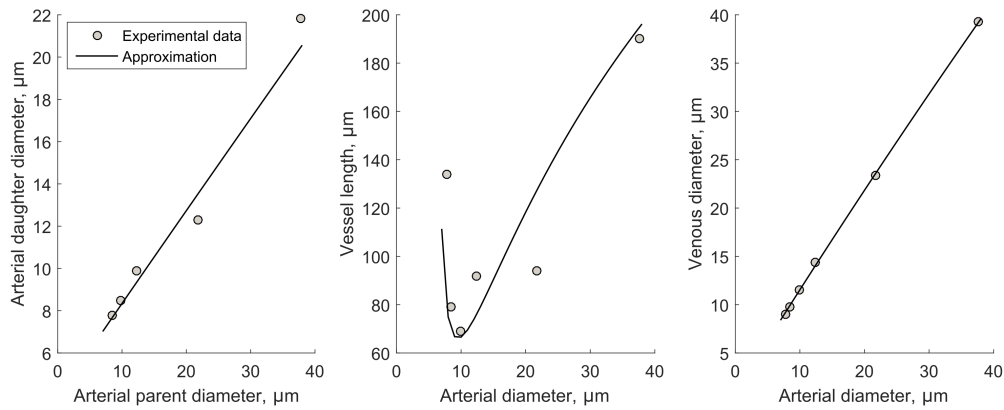

Fig. 1. Approximations (black curves) and experimental data ${ }^{26}$ (gray circles) for structure generation. (Left panel) Daughter diameter as a function of parent-vessel diameter for the arterial tree. (Center panel) Vessel length as a function of its diameter. (Right panel) Venous diameter compared to arteriolar diameter in the same branching level.

We have generated two networks to study the effects of the myogenic response gradient and vascular structure asymmetry. Following Murray's law for diameter, the symmetrical vascular structure can only have one set of arterial side diameters $\left(D_{p}^{3}=\right.$ $2\left(D_{d}^{3}\right)$, where $D_{p}$ and $D_{d}$ are parent and daughter vessels diameter, respectively) for the given size of the largest artery. For an asymmetrical structure, the diameter of the first daughter vessel was calculated according to real data approximation, whereas Murray's law was used for the second one. Vessel length and venous side diameters were calculated according to approximations. Both symmetrical and asymmetrical networks were generated using a diameter equal to $50 \mu \mathrm{m}$ for the largest arterial vessel and of $\approx 8 \mu \mathrm{m}$ for capillaries. The resulting networks consisted of 382 and 541 vessels for symmetrical and asymmetrical networks, respectively.

For each type of vessel, the initial wall thickness was included as follows:

1. Capillaries: We assume a capillary wall thickness of $0.5 \mu \mathrm{m}^{28}$ and a diameter of approximately $6 \mu \mathrm{m}$.

2. Arterioles: From the experimental data, ${ }^{29}$ the wall thickness of arterioles, $h_{a}$, is approximated as $h_{a}=0.1319 \cdot r_{i}+1.352 \mu \mathrm{m}$.

3. Venules: Similarly, from the experimental data, ${ }^{29}$ the wall thickness of venules, $h_{v}$, is approximated as $h_{v}=0.08858 \cdot r_{i}+1.045 \mu \mathrm{m}$.

Note that, for the data used to generate the networks, ${ }^{26}$ the inner radius is known while the tone giving rise to that radius in the individual vessel is unknown. Since the 
purpose here is merely to generate a network structure, we assume for simplicity that the experimentally derived inner radius, $r_{i 0}$, at $t=0$ represents the slack state of the vessel, hence $r_{m \text {, slack }}=r_{i 0}+\left.h\right|_{t=0} / 2$. This value for $r_{m}$, slack was used as the initial condition.

Although the wall dynamics of each vessel is processed independently, all vessels influence one another through intravascular pressure. Based on the rate of change in pressure in each node, $P_{n},{ }^{16,30,31}$ can be expressed as:

$$
C \frac{d P_{n}}{d t}=\frac{P_{c n 1}-P_{n}}{R_{c v 1}}-\frac{P_{n}-P_{c n 2}}{R_{c v 2}}-\frac{P_{n}-P_{c n 3}}{R_{c v 3}},
$$

where $C$ expresses the compliance of the system, $R=1 / C_{h d r}$ is the hemodynamical resistance, $C_{h d r}=\pi r_{i}^{4} / 8 \eta l$ is the hemodynamical conductance where $\eta$ is the blood viscosity, indexes $c n 1, c n 2$, and $c n 3$ correspond to the $1^{\text {st }}, 2^{\text {nd }}$, and $3^{\text {rd }}$ adjacent connected nodes, indexes $c v 1, c v 2$, and $c v 3$ correspond to the $1^{\text {st }}, 2^{\text {nd }}$, and $3^{r d}$ vessels connected to the node in question, and where the $1^{\text {st }}$ is the inlet vessel, and the $2^{\text {nd }}$ and $3^{r d}$ are outlet vessels. A large value of $C$ corresponds to a pronounced ability of the network to absorb rapid pressure fluctuations. In contrast, if $C$ is small, fluctuations in inlet pressure $\left(P_{\text {in }}\right)$ or outlet pressure $\left(P_{\text {out }}\right)$ will transmit instantaneously throughout the network.

Before each simulation, the system was run to steady state for the current values of control parameters ( $P_{\text {in }}, P_{\text {out }}$, and vascular network and vessel structure). This was achieved by first solving the system of Equation (16). Next, the system describing each individual vessel was solved for the obtained node pressures regarding the conditions $d \varepsilon / d t=0, d \Psi / d t=0$, and $d \Omega / d t=0$. Node pressures were then recalculated and so forth. Iterations were repeated until the above condition was fulfilled (relative change less than $1 \cdot 10^{-10}$ per iteration round).

\section{Network properties: simulation results}

\subsection{Myogenic response}

We simulate three different situations (Fig. 2) and display vessel size as the relative radius, i.e., $r_{m} / r_{m \text {,slack }}$ for vessels of different type and diameter. In all cases, $P_{i n}$ and $P_{\text {out }}$ are increased symmetrically in steps of $1 \mathrm{~Pa} / \mathrm{s}$ over $20000 \mathrm{~s}$ starting from $0.5 \mathrm{kPa}$ :

1. Passive model (Fig. 2a): For the passive model, the tone in vessels was set to 0.00001 . In response to an increase in transmural pressure, all vessels display similar behavior, characterized by passive distension.

2. Model without a myogenic response gradient (Fig. 2b): Approximating functions func $_{1}\left(r_{i 0}\right)$ and $\operatorname{func}_{2}\left(r_{i 0}\right)$ are equal to 1.0. The difference in the pressure range characterizing the curves in this situation is therefore due only to the differences in vessel morphology and not to the variable sensitivity of SMC to circumferential wall stress. Again, venous vessels and capillaries dilate with increasing pressure due to absence 
of an active myogenic response. Note that the largest vessel (No. 1 in Fig. 2, with a radius of $80 \mu \mathrm{m}$ ) in this situation has the shortest active range. This is caused by the relative wall thickness being smaller in larger vessels. An increase in intravascular pressure will therefore cause a faster increase in circumferential stress, and hence, in activation, the larger the vessel. After maximal activation is reached, the vessel will experience forced dilation following a further increase in pressure.

3. Model with a myogenic response gradient (Fig. 2C): The maximum myogenic response is displayed by arterioles with a radius of $22 \mu \mathrm{m}$, while for smaller and larger arteries the response is less pronounced. Smaller vessels dilate earlier than large ones. As stated before, venous vessels and capillaries show distension in response to increasing pressure.

\subsection{Filtration}

To study the ability of a microvascular network to filtrate pressure alterations in relation to the resulting flow and pressure at the capillary level, we used blood pressure data from a rat aorta as the inlet pressure in the model.

In brief, arterial blood pressure was continuously recorded in a conscious, freely moving male Sprague Dawley rat (Taconic Biosciences, Inc., Ejby, Denmark) with a radiotelemetric transmitter. The transmitter (model PA-C40; Data Science International, St. Paul, MN) was surgically implanted into the abdominal aorta of the rat at least ten days prior to data collection, with the pressure-sensing catheter placed just below the renal arteries. Blood pressure was collected with a frequency of $500 \mathrm{~Hz}$.

For the present purpose, we selected a single continuous recording of around 30 minutes taken during the night - the active period for nocturnal animals - when arterial blood pressure level fluctuations are more pronounced than in the daytime. Since the experimental data was obtained from the aorta but the present model is concerned with much smaller vessels, a rescaling was performed to reach a realistic network inlet pressure (corresponding to the physiological pressure of an arteriole around 160 microns in diameter). The scaling coefficient was calculated as the ratio between the average pressure observed in the experiment and expected inlet pressure of the network $\left(P_{i n}=7 \mathrm{kPa}\right.$ ). Each pressure value in the time series was divided by this coefficient to rescale the experimental signal while keeping its shape.

As discussed previously (Fig. 2), we consider three types of models with different myogenic responsiveness and compare their filtering properties (Fig. 3). In all cases, network outlet pressure was kept constant at $\left(P_{\text {out }}=1.7 \mathrm{kPa}\right)$. Figure $3 a$ shows the scaled time series.

In the case of a completely passive network (Fig. 3b), the vessels stand distended at all pressures derived from the pressure time series, with the transmural pressure being carried mainly by the stretched passive elastic material of the wall. Pressure fluctuations have therefore relatively little effect on radius, giving the network an overall "stable" appearance. However, the passive network does not shield the capillary bed from hyperperfusion and a potentially deleterious pressure. 
a)

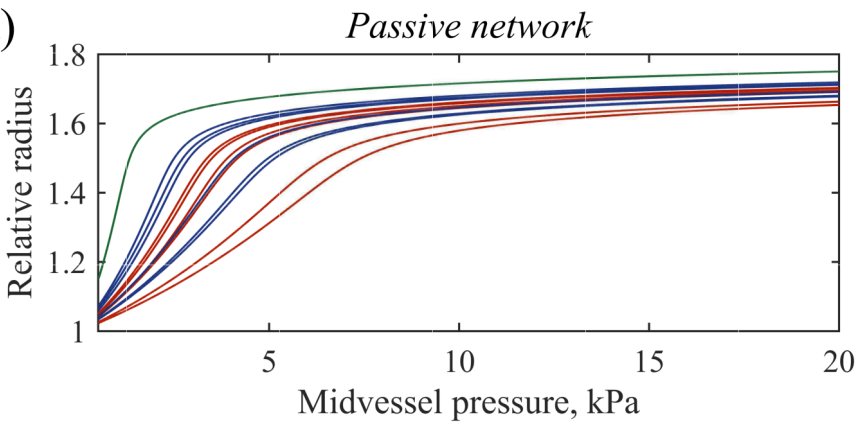

b)

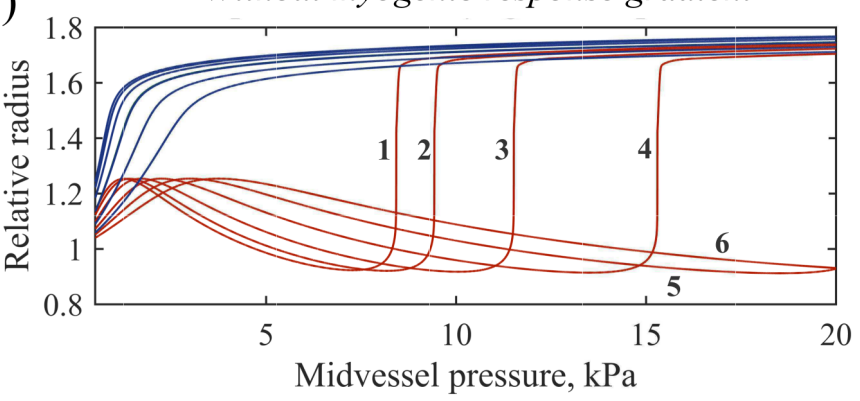

c)

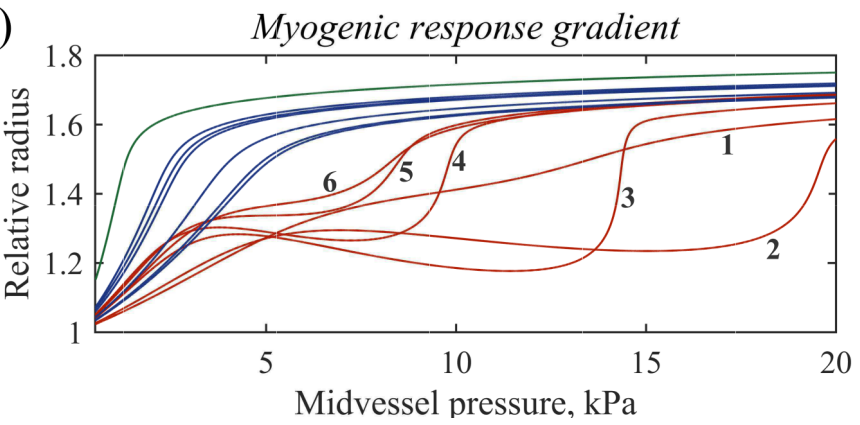

Fig. 2. The response of vessels of different diameter and type to increasing pressure. (a) Passive system, (b) model without a myogenic response gradient, and (c) model with a myogenic response gradient. $P_{\text {in }}$ and $P_{\text {out }}$ are increased symmetrically from $500 \mathrm{~Pa}$ to $20.5 \mathrm{kPa}$ in steps of $1 \mathrm{~Pa}$. Different colors depict arterial vessels (red), venular vessels (blue), and capillaries (green). 1: $r_{i 0}=80 \mu m$, 2: $r_{i 0}=38.8 \mu m$, 3: $r_{i 0}=18.6 \mu m$, 4: $r_{i 0}=9.6 \mu m$, 5: $r_{i 0}=5.65 \mu m, 6: r_{i 0}=$ $3.9 \mu \mathrm{m}$. 
a)

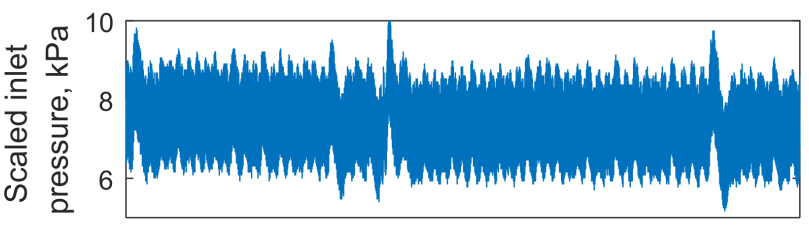

b)

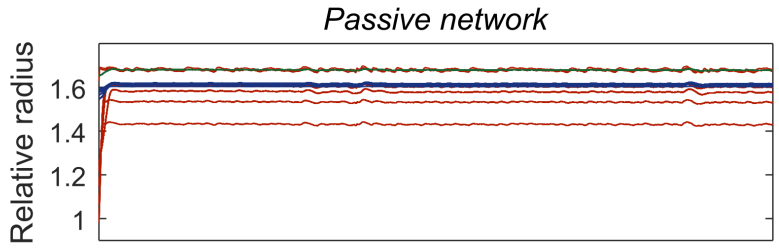

c)

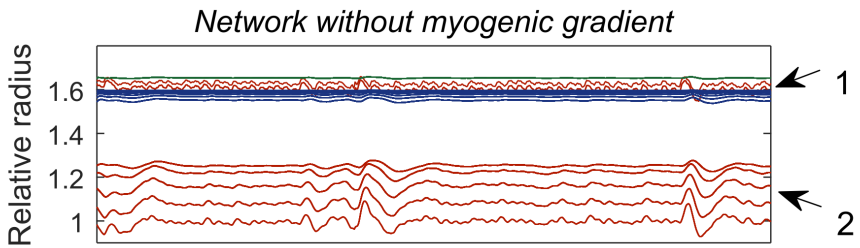

d)

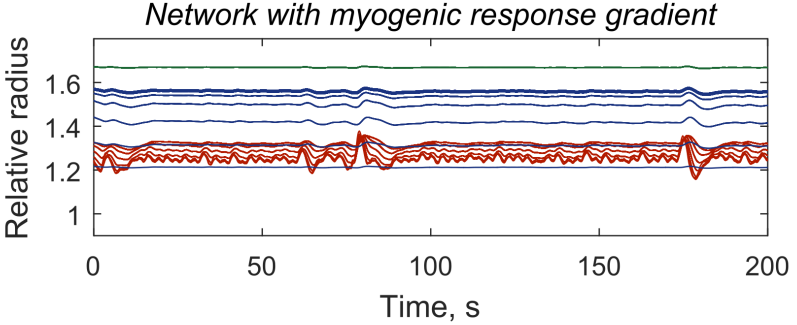

Fig. 3. Relative radii in a 382-vessel symmetrical network. (a) Rescaled network inlet pressure based on experimental data from rat aorta, (b) Passive network, (c) network without a myogenic response gradient, and (d) network with a myogenic response gradient. Different colors indicate arteriolar vessels (red), venular vessels (blue) and capillaries (green). 1: Largest arterial $r_{i 0}=50$ and $39 \mu m$ that dilate earlier than 2: smaller vessels. 
In the network without a myogenic gradient the situation is somewhat different (Fig. 3c). As indicated, the first two generations of vessels have experienced forced dilatation despite maximal activation, whereas generations of vessels with smaller diameters are constricted. Due to this profound constriction, they have a shielding effect on the downstream network.

Figure $3 \mathrm{~d}$ shows the behavior of different network vessels in the presence of a myogenic response gradient. In this case, the whole arteriolar network participates both in the dampening of pressure fluctuations as in the regulation of downstream pressure.

To further investigate how myogenic properties influence network filtration properties, a Fourier analysis (fast Fourier transform, FFT) is applied to the flow in selected vessels. The resulting power spectra shown in Figure 4 provide information about the distribution of the signal energy, i.e., amplitude of oscillations as a function of frequencies. 32

Note that, in contrast to the other panels in Figure 4, Figure 4a is a FFT of the inlet pressure signal shown in Figure 3a, not one of flow. There are a few well-pronounced peaks at $0.36 \mathrm{~Hz}, 1-1.7 \mathrm{~Hz}, 5 \mathrm{~Hz}, 10 \mathrm{~Hz}$ and $15 \mathrm{~Hz}$ in the input signal which are also reflected in the flow signal, especially from the largest arteries of the network (Fig. 4b and Fig. 4c). Low-frequency oscillations $(0.36 \mathrm{~Hz})$ possibly reflect Mayer waves. It is known that the $5 \mathrm{~Hz}$ peak is associated with the pulse wave frequency. The next doubled and tripled frequencies are harmonics of the pulse wave frequency. These peaks decrease rapidly in the power spectrum for smaller arterioles, disappearing altogether in the venous vessels. Only the low-frequency oscillations persist on the venous side of the network. Thus, independently of the myogenic properties, the system acts as a low-pass filter.

Inspection of the curves, however, reveals some differences. At the level of the larger arterioles, having a myogenic response gradient provides a stronger dampening effect on oscillations in flow throughout the frequency range compared to that of the other two networks. At the postcapillary level, the models with and without a myogenic response seem to display similar efficiency. Passive networks have the worst filtering properties on all levels.

\subsection{Myogenic response gradient as a protective mechanism}

In Figure 5 we compare the behaviour of the three models with different myogenic responsiveness (passive, equal maximal, or gradient) along the network. The left side of the figure represents the symmetrical network; the right side represents the asymmetrical case. Note that the asymmetrical network has more vessels than the symmetrical network (541 versus 382, respectively). This is caused by symmetry, which in turn minimizes the number of vessels.

To investigate their autoregulatory properties as a whole, the networks are exposed to a ramping inlet pressure while the outlet pressure is kept constant. As expected, in completely passive networks blood flow rises in almost linear proportion 
a)

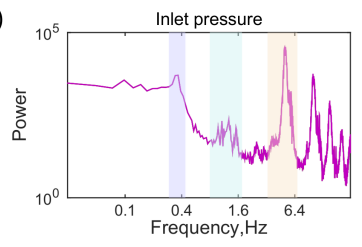

c)

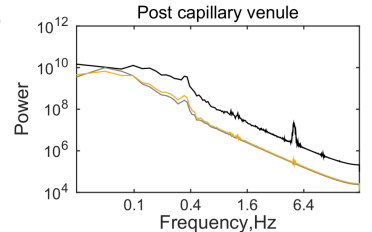

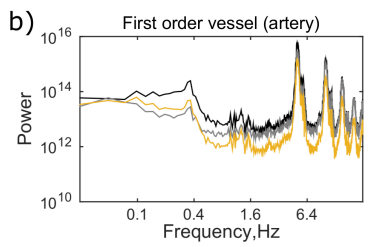

d) 10

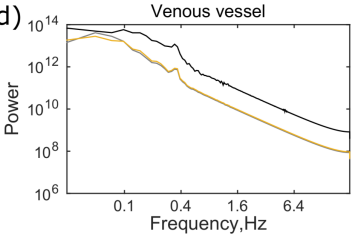

Network without myogenic response gradien Network with myogenic response gradient

Fig. 4. Log-log plots of power spectra for experimental inlet pressure (purple) and for flow time series in different vessels in the passive network (black), the network with equal and maximal myogenic response (gray), and the network with a myogenic response gradient (yellow).

to increases in perfusion pressure. In contrast, over a certain range of pressures, both of the two active networks tend to maintain a more stable flow. The model with a myogenic response displays the best flow regulation. This may be explained by the fact that, in the model without a myogenic gradient, large vessels dilate first, leading to a rapid increase in flow.

Comparing the autoregulatory properties of symmetrical versus asymmetrical networks, asymmetry leads to even better regulation for all the networks, including the passive one. It is caused by the system having more vessels, and consequently, larger resistance and better flexibility in terms of myogenically-governed blood flow control.

\section{Discussion}

Myogenic response is a complex adjusting mechanism which regulates vessel wall tone in response to the stretching of SMC due to changing intravascular pressure. Given that our model is based on vascular wall mechanics rather than cellular physiology, we are able to introduce the myogenic response and its gradient only by means of mathematical descriptive formulation, reproducing the myogenic response and its gradient in a pseudo steady state (Fig. 2c).

It is known that the myogenic response gradient not only increases with decreasing arterial diameter, decreasing again at the smallest arterioles, but also regulates vessel wall tone within different pressure ranges throughout the arterial network. ${ }^{4}$ Thus, finding the correct myogenic response gradient formulation, one which takes into account these features, is no trivial task. A desirable gradient was achieved by modifying all three types of vessel wall stresses. 

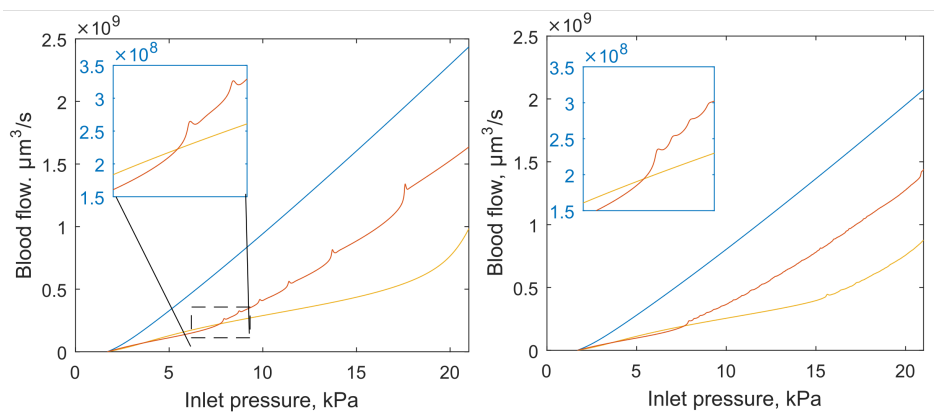

Fig. 5. Comparison of auto-regulatory features of a symmetrical network (left) and asymmetrical networks (right). The networks are exposed to a ramping inlet pressure of 1.7 to $22 \mathrm{kPa}$ in steps of $10 \mathrm{~Pa}$. Outlet pressure is kept constant at $1.7 \mathrm{kPa}$. Curves are shown for the three models: passive (black), without a myogenic response gradient (gray), and with a myogenic response gradient (yellow).

The modification of SMC sensitivity to stress influences active stress development while SMC contract and seems to be a natural mechanism that allows the emergence of the myogenic response gradient.The modification of passive and cytoskeletal stresses is also included in the network with a myogenic response gradient. However, at a given pressure range, while arterioles show significant myogenic activity, most of the total stress in the network will be generated by contraction of SMC. Hence, it is possible to compare a passive network and a network without myogenic response gradient, where these modifications are not included, with a myogenic response gradient network where the impact of cytoskeletal and passive stress is less than than the impact of active stress. The impact of the cytoskeletal and passive stress components becomes higher when vessels are forced dilated. For this reason, we find that the present mathematical formulation of the myogenic response gradient is reasonable at the moment, although it shall be improved in the future.

Our main findings were:

1. Networks with a realistic distribution of the relative wall thickness reacted inadequately to changes in perfusion pressure if all vessels had the same sensitivity to wall stress and acted with the same force development (i.e., the model without a myogenic response gradient).

2. A gradient in stress sensitivity allowing larger upstream vessels to react less vigorously but over a broader pressure range compared to smaller, more downstream vessels was associated with a physiologically realistic behavior where the vessels of the arteriolar network acted concertedly to changes in pressure.

We observed that the network is able to dampen oscillations in all cases, including in the complete absence of tone. There are several reasons for this. First, for the calculation of node pressures, a formulation derived from the classical wind-kessel vessel model was applied (Eq. 16). This allows for a transient volume change in a node following a change in pressure. This "reservoir" function introduces a dampening effect 
on the pressure oscillations that follow from fluctuations in perfusion pressure. In this formulation, a step change in inlet pressure will propagate throughout the network at a rate determined by compliance. Secondly, as outlet pressure is fixed, the network is under all circumstances forced to reduce pressure along the different flow paths to eventually match the outflow pressure regardless of the material properties of the vessels. Taken together, we therefore expected, and indeed found, that the network dampens oscillations in inlet pressure both in the complete absence of tone and under the two different kinds of myogenic reactivity. The efficiency of this process varies, however, with the passive network being the least efficient of all. For the two kinds of myogenic reactivity, the one without a myogenic response gradient appears to dampen oscillations at the capillary level as efficiently as the model with a myogenic response gradient. This is due to an inadequate response where the most upstream vessels experience forced dilation and the precapillary vessels are in a state of profound constriction. Hence, in the present formulation, the network with a myogenic response gradient exerts the most adequate response (Fig. 3-Fig. 4).

A main function of microcirculation is not only its ability to deliver a graded flow in response to the changing metabolic needs of surrounding tissues, but also to maintain a stable flow in the face of fluctuating perfusion pressure. In that context, the present analysis points to a fundamental problem when the network lacks a myogenic response gradient but rather displays a uniform stress sensitivity in all vessels. Since the walls of larger, more upstream vessels are relatively thinner, ${ }^{29}$ and since such vessels are exposed to higher pressure due to their more upstream position, they will, due to the law of Laplace (Eq. 7), inevitably be exposed to higher stress. If the stress level to which they react myogenically and the force they are able to develop is not adjusted accordingly, the consequences will be as shown in Figures 3-5: upstream vessels will experience high stress, and hence, a high level of activation, and will even at moderate pressures experience forced dilation, whereas at the network level where vessels are able to withstand the pressure they will be in a state of deep constriction. This behavior is not compatible with either a graded regulation of flow or a smooth reaction to an increase in perfusion pressure (Fig. 5). Besides, since the ability of the SMC to develop force is a bell-shaped function of SMC length, the vessel is at risk of getting locked up in the dilated state and remain there even when pressure is lowered, simply because maximal force development is smaller and stress is larger the more stretched the vessel is. On the other hand, the presence of a myogenic response gradient circumvents these problems. In that case, the stress optimum as regards the operation of the individual vessel is matched to the physiological pressure and vascular anatomy prevailing at that position in the network. Larger, more upstream vessels exposed to a higher average pressure and with a relatively thinner wall must operate at a higher stress level. Such vessels also generally display myogenic reactivity over a broader pressure range, ${ }^{5}$ which also fits with these vessels being exposed to the largest pressure fluctuations. Due to the action of these upstream vessels, more downstream vessels receive a reduced pressure with dampened oscillations, and hence can operate over a more narrow pressure range but with a steeper 
slope on the myogenic curve to efficiently fine tune pressure and flow as the blood approaches the capillary bed.

On a network level, the autoregulation curve therefore becomes smooth, and the ability to react to stimuli other than pressure, e.g., changes in metabolism, will remain intact over a broad range of pressures.

Parameter sensitivity tests for $\mathrm{k} 2\left(k_{2}=0.0075,0.0375,0.375\right.$, and 0.75$)$ and $\mathrm{C}(C=$ $2500,3000,30000$ ) did not show significantly different results, except expected quantitative changes in filtration properties. When $C$ is set to values below 2500 , the system becomes unstable.

In conclusion, the network with a myogenic gradient shows advantages regarding the physiological function of regulating flow in a bifurcating network. The whole arteriolar network participates in this regulation, with each vessel operating around a pressure that corresponds to its position in the network. The flow-autoregulation curve therefore becomes smooth and the individual vessel is not exposed to excessive stress. At the same time, with this kind of reactivity, the network avoids the risk of upstream closure and/or forced dilation which, in relation to tissue metabolism, potentially leads to inappropriate flow.

\section{Acknowledgements}

The author is grateful to Olga Sosnovtseva, Jens Christian Brings Jacobsen, and Dmitry Postnov Jr. for their helpful discussions and suggestions, and to Morten Lund for contributing with the time series data. This work was made possible by a Marie Curie grant from the European Commission in the framework of the REVAMMAD ITN (Initial Training Network), Project number 316990.

\section{Appendix}

Approximating functions used for the generation of symmetrical and asymmetrical vascular networks:

1. Dependence of daughter vessel diameter $d_{d a u g}$ on parent vessel diameter $d_{\text {par }}$ :

$$
d_{\text {daug }}=p_{11} \cdot d_{\text {par }}^{2}+p_{12} \cdot d_{\text {par }}+p_{13},
$$

where $p_{11}=-0.000164, p_{12}=0.4442, p_{13}=3.916 . d_{\text {par }}$ and $d_{\text {daug }}$ are measured in $\mu m$.

2. Dependence of vessel length $l$ on vessel diameter $d$ :

$$
l=\frac{p_{21} \cdot d^{4}+p_{22} \cdot d^{3}+p_{23} \cdot d^{2}+p_{24} \cdot d+p_{25}}{d^{2}+q_{21}+q_{22}},
$$

where $p_{21}=-0.002145, p_{22}=1.307, p_{23}=326.5, p_{24}=-3970, p_{25}=1.587 \mathrm{e}+04, q_{21}$ $=20.07, q_{22}=$-148.9. $l$ and $d$ are measured in $\mu \mathrm{m}$. 
Table 1. Table of default parameters

\begin{tabular}{|l|l|l|}
\hline Parameter & Value & Units \\
\hline$\mu$ & 0.0035 & $P a \cdot s$ \\
\hline$C$ & $3 \cdot 10^{-15}$ & $m^{3} / P a$ \\
\hline$f_{p}, f_{a}$ and $f_{c}$ & $1 / 3$ & - \\
\hline$C p_{1}^{*}$ & $5.5 \cdot 10^{4}$ & $N / m^{2}$ \\
\hline$a p_{1}$ & 1.5 & - \\
\hline$C p_{2}^{*}$ & 0.0186 & $N / m^{2}$ \\
\hline$a p_{2}$ & 24 & - \\
\hline$l_{r e f}$ & $80 \cdot 10^{-6}$ & $m$ \\
\hline$l_{a o p t}$ & $1.0618 \cdot 10^{-4}$ & $m$ \\
\hline$\sigma_{a m_{o p t}}^{*}$ & $2.5 \cdot 10^{5}$ & $N / m^{2}$ \\
\hline$b_{a}$ & $4.5868 \cdot 10^{-5}$ & $m$ \\
\hline$l_{c 0}$ & $1.1892 \cdot 10^{-4}$ & $m$ \\
\hline$\sigma_{c 0}^{*}$ & $2.5 \cdot 10^{5}$ & $N / m^{2}$ \\
\hline$b_{c}$ & 30 & $1 / m$ \\
\hline$\sigma_{50}$ & $3 \cdot 10^{4}$ & $N / m^{2}$ \\
\hline$h c$ & 3 & - \\
\hline$k_{1}$ & $15 \cdot 10^{-6}$ & $m^{2} /(N \cdot s)$ \\
\hline$k_{2}$ & 0.075 & $1 / s$ \\
\hline$k_{3}$ & 56.25 & $1 /(m \cdot s)$ \\
\hline$\Psi_{v}$ & 0.05 & - \\
\hline$\Psi_{c}$ & 0.01 & - \\
\hline$N$ & $2^{11}$ & - \\
\hline & & \\
\hline
\end{tabular}

3. Dependence of venous diameter $d_{v}$ on corresponding arterial diameter $d_{a}$ :

$$
d_{v}=p_{31} \cdot d_{a}^{2}+p_{32} \cdot d_{a}+p_{33}
$$

where $p_{11}=-0.0005603, p_{12}=1.127, p_{13}=1.335 . d_{a}$ and $d_{v}$ are measured in $\mu \mathrm{m}$. Approximating functions used to generate the myogenic response gradient:

$$
\begin{aligned}
& \operatorname{func}_{1}\left(r_{i 0}\right)=\sqrt{r_{i 0}} \cdot e^{\frac{-r_{i 0}}{\text { par }_{1}}} \cdot \operatorname{par}_{2}, \\
& \operatorname{func}_{2}\left(r_{i 0}\right)=\frac{\sqrt{r_{i 0}} \cdot e^{\frac{-r_{i 0}}{\text { par }_{1}}} \cdot \operatorname{par}_{2} \cdot\left(\bar{\sigma}^{h c}+\sigma_{50}^{3}\right)}{\bar{\sigma}^{h c}+\frac{\sigma_{50}^{3}}{\text { par }_{3} \cdot e^{-p a r_{4} \cdot r_{i 0}}+\text { par }_{5}}},
\end{aligned}
$$

where $r_{i 0}$ is initial inner vessel radius, $\operatorname{par}_{1}, \operatorname{par}_{2}$, par $_{3}$, par $_{4}$, and $\operatorname{par}_{5}$ are parameters that define the shapes of the curve $f u n c_{1}$ and the surface $f u n c_{2}$. 
Table 2. Parameters of the approximating functions

\begin{tabular}{|l|l|l|}
\hline Parameter & Value & Units \\
\hline par $_{1}$ & 45 & $\mu m$ \\
\hline par $_{2}$ & 0.28 & $1 / \sqrt{\mu m}$ \\
\hline par $_{3}$ & 1.16 & - \\
\hline par $_{4}$ & 0.08 & $1 / \mu m$ \\
\hline par $_{5}$ & 0.182 & - \\
\hline
\end{tabular}

\section{References}

1. Bayliss W. On the local reactions of the arterial wall to changes of internal pressure. J Physiol, 1902;28(3): 220-231. Available from: https://www.ncbi.nlm.nih.gov/pubmed/16992618.

2. Osol G, Brekke J, McElroy-Yaggy K, Gokina N. Myogenic tone, reactivity, and forced dilatation: a threephase model of in vitro arterial myogenic behavior. Am J Physiol Heart Circ Physiol, 2002;283(6): H2260-H2267. Available from: https://www. ncbi.nlm.nih.gov/pubmed/12388265. doi: 10.1152/ ajpheart.00634.2002.

3. Clifford P. Local control of blood flow. Adv Physiol Educ, 2011;35(1): 5-15. Available from: https://www. ncbi.nlm.nih.gov/pubmed/21385995. doi: 10.1152/advan.00074.2010.

4. Davis M, Hill M. Signaling mechanisms underlying the vascular myogenic response. Physiol Rev, 1999;79(2): 387-423. Available from: https://www.ncbi.nlm.nih.gov/pubmed/10221985.

5. Davis M. Myogenic response gradient in an arteriolar network. Am. J. Physiol, 1993;264(6 Pt 2): H2168H2179. Available from: https://www.ncbi.nlm.nih.gov/pubmed/8322948.

6. Jeppesen P, Sanye-Hajari J, Bek T. Increased blood pressure induces a diameter response of retinal arterioles that increases with decreasing arteriolar diameter. Invest Ophthalmol Vis Sci, 2007;48(1): 328-331. Available from: https : / www . ncbi . nlm . nih . gov / pubmed / 17197550. doi: 10.1167/iovs.06-0360.

7. Schubert R, Mulvany M. The myogenic response: established facts and attractive hypotheses. Clin Sci (Lond), 1999;96(4): 313-326. Available from: https://www.ncbi.nlm.nih.gov/pubmed/10087237.

8. Yang J, Jr. JC, Bryan R, Robertson C. The myogenic response in isolated rat cerebrovascular arteries: smooth muscle cell model. Med Eng Phys, 2003;25(8): 691-709. Available from: https://www.ncbi. nlm.nih.gov/pubmed/12900184.

9. Yang J, Jr. JC, Bryan R, Robertson C. The myogenic response in isolated rat cerebrovascular arteries: vessel model. Med Eng Phys, 2003;25(8): 711-717. Available from: https://www.ncbi.nlm.nih.gov/ pubmed/12900185.

10. Carlson B, Secomb T. A theoretical model for the myogenic response based on the length-tension characteristics of vascular smooth muscle. Microcirculation, 2005;12(4): 327-338. Available from: https://www.ncbi.nlm.nih.gov/pubmed/16020079. doi: 10.1080/10739680590934745.

11. Jacobsen J, Mulvany M, Holstein-Rathlou N. A mechanism for arteriolar remodeling based on maintenance of smooth muscle cell activation. Am J Physiol Regul Integr Comp Physiol, 2008;294(4): R1379R1389. Available from: https://www.ncbi.nlm.nih.gov/pubmed/18184768. doi: 10.1152/ajpregu.00407. 2007.

12. Cornelissen A, Dankelman J, VanBavel E, Stassen H, Spaan J. Myogenic reactivity and resistance distribution in the coronary arterial tree: a model study. Am J Physiol Heart Circ Physiol, 2000;278(5): H1490-H1499. Available from: https://www.ncbi.nlm.nih.gov/pubmed/10775126.

13. Hacking W, VanBavel E, Spaan J. Shear stress is not sufficient to control growth of vascular networks: a model study. Am J Physiol, 1996;270(1 Pt 2): H364-H375. Available from: https://www.ncbi.nlm.nih. gov/pubmed/8769773. 
14. Jacobsen JB, Gustafsson F, Holstein-Rathlou N-H. A model of physical factors in the structural adaptation of microvascular networks in normotension and hypertension. Physiol Meas, 2003;24(4): 891912. Available from: https://www.ncbi.nlm.nih.gov/pubmed/14658781.

15. Guidoboni G, Harris A, Cassani S, Arciero J, Siesky B, Amireskandari A, et al. Intraocular pressure, blood pressure, and retinal blood flow autoregulation: a mathematical model to clarify their relationship and clinical relevance. Invest Ophthalmol Vis Sci, 2014;55(7): 4105-4118. Available from: https: //www.ncbi.nlm.nih.gov/pubmed/24876284. doi: 10.1167/iovs.13-13611.

16. Postnov D, Postnov D, Marsh D, Holstein-Rathlou N, Sosnovtseva O. Dynamics of Nephron-Vascular Network. Bull Math Biol, 2012;74(12): 2820-2841. Available from: https://www.ncbi.nlm.nih.gov/ pubmed/23081729. doi: 10.1007/s11538-012-9781-6.

17. Jacobsen JB, Hornbech M, Holstein-Rathlou N-H. Significance of microvascular remodelling for the vascular flow reserve in hypertension. Interface Focus, 2011;1(1): 117-131. Available from: https://www. ncbi.nlm.nih.gov/pubmed/22419978. doi: 10.1098/rsfs.2010.0003.

18. Pries A, Secomb T, Gaehtgens P. Structural adaptation and stability of microvascular networks: theory and simulations. Am J Physiol, 1998;275(2 Pt 2): H349-H360. Available from: https://www.ncbi.nlm. nih.gov/pubmed/9683420.

19. Pries A, Cornelissen A, Sloot A, Hinkeldey M, Dreher M, Höpfner M, et al. Structural Adaptation and Heterogeneity of Normal and Tumor Microvascular Networks. PLoS Comput Biol, 2009;5(5): e1000394. Available from: https://www.ncbi.nlm.nih.gov/pmc/articles/PMC2682204/. doi: 10.1371/journal.pcbi. 1000394.

20. Fry B, Roy T, Secomb T. Capillary recruitment in a theoretical model for blood flow regulation in heterogeneous microvessel networks. Physiol Rep, 2013;1(3): e00050. Available from: https://www.ncbi. nlm.nih.gov/pubmed/24040516. doi: 10.1002/phy2.50.

21. VanBavel E, Tuna B. Integrative Modeling of Small Artery Structure and Function Uncovers Critical Parameters for Diameter Regulation. PLoS One, 2014;9(1): e86901. Available from: https://www.ncbi. nlm.nih.gov/pubmed/24497993. doi: 10.1371/journal.pone.0086901.

22. Feldberg R, Colding-Jørgensen M, Holstein-Rathlou N. Analysis of interaction between TGF and the myogenic response in renal blood flow autoregulation. Am J Physiol, 1995;269(4 Pt 2): F581-F593. Available from: https://www.ncbi.nlm.nih.gov/pubmed/7485545.

23. Kuo L, Arko F, Chilian W, Davis M. Coronary venular responses to flow and pressure. Circ Res, 1993;72(3): 607-615. Available from: https://www.ncbi.nlm.nih.gov/pubmed/8431988.

24. Dörnyei G, Monos E, Kaley G, Koller A. Myogenic responses of isolated rat skeletal muscle venules: modulation by norepinephrine and endothelium. Am J Physiol, 1996;271(1 Pt 2): H267-H272. Available from: https://www.ncbi.nlm.nih.gov/pubmed/8760184.

25. Hall C, Reynell C, Gesslein B, Hamilton N, Mishra A, Sutherland B, et al. Capillary pericytes regulate cerebral blood flow in health and disease. Nature, 2014;508(7494): 55-60. Available from: https:// www.ncbi.nlm.nih.gov/pubmed/24670647. doi: 10.1038/nature13165.

26. Schröder S, Brab M, Schmid-Schönbein GW, Reim M, Schmid-Schönbein H. Microvascular network topology of the human retinal vessels. Fortschr Ophthalmol, 1990;87(1): 52-58. Available from: https: //www.ncbi.nlm.nih.gov/pubmed/2323698.

27. Postnov DD, Marsh DJ, Postnov DE, Braunstein TH, Holstein-Rathlou N-H, Martens EA, et al. Modeling of kidney hemodynamics: Probability-based topology of an arterial network. PLoS Comput Biol, 2016;12(7): e1004922. Available from: https://www.ncbi.nlm.nih.gov/pmc/articles/PMC4957782/. doi: 10.1371/journal.pcbi.1004922.

28. Sosula L. Capillary radius and wall thickness in normal and diabetic rat retinae. Microvasc Res, 1974;7(2): 274-276. Available from: https://www.ncbi.nlm.nih.gov/pubmed/4274673.

29. Gore RW. Pressures in cat mesenteric arterioles and capillaries during changes in systemic arterial blood pressure. Circ Res, 1974;34(4):581-591. Available from: https://www.ncbi.nlm.nih.gov/pubmed/ 4826932.

30. Frank O. The basic shape of the arterial pulse. First treatise: mathematical analysis. 1899. J Mol Cell Cardiol, 1990;22(3): 255-277. Available from: https://www.ncbi.nlm.nih.gov/pubmed/21438422. 
31. Marsh D, Sosnovtseva O, Mosekilde E, Holstein-Rathlou N-H. Vascular coupling induces synchronization, quasiperiodicity, and chaos in a nephron tree. Chaos, 2007;17(1): 015114. Available from: https: //www.ncbi.nlm.nih.gov/pubmed/17411271. doi: 10.1063/1.2404774.

32. Numerical recipes in C (2nd ed.): the art of scientific computing. Cambridge University Press New York, 1992; chap. Power Spectrum Estimation Using the FFT. 